\title{
Superconducting Properties of $\mathrm{Mo}_{2} \mathrm{Re}_{3} \mathrm{~B}_{x}-\mathrm{Mo}_{3} \mathrm{Re}_{2} \mathrm{~B}_{x}$ Eutectic
}

\author{
B. Andrzejewski ${ }^{a, b, *}$, E. Gullmeau ${ }^{b}$, A. KowalczyK ${ }^{a}$ \\ AND W. JURGA ${ }^{c}$ \\ ${ }^{a}$ Institute of Molecular Physics, Polish Academy of Sciences \\ Smoluchowskiego 17, 60-179 Poznań, Poland \\ ${ }^{b}$ Laboratoire CRISMAT, UMR 6508 CNRS-ENSICAEN \\ 6, Boulevard du Maréchal Juin, 14050 Caen Cedex, France \\ ${ }^{c}$ Institute of Physics, A. Mickiewicz University \\ Umultowska 85, 61-614 Poznań, Poland
}

\begin{abstract}
We synthesized, for the first time, superconducting eutectic composed of two new superconducting phases, namely of $\mathrm{Mo}_{2} \mathrm{Re}_{3} \mathrm{~B}_{x}$ and of $\mathrm{Mo}_{3} \mathrm{Re}_{2} \mathrm{~B}_{x}$ (where $x \approx 1$ ) and investigated its basic transport, magnetic, and microwave properties. The transition temperatures $T_{\mathrm{c}}$ were equal to $6.6 \mathrm{~K}$ and to $8.7 \mathrm{~K}$ for $\mathrm{Mo}_{3} \mathrm{Re}_{2} \mathrm{~B}_{x}$ and $\mathrm{Mo}_{2} \mathrm{Re}_{3} \mathrm{~B}_{x}$ compound, respectively. The phases present in the eutectic were identified by means of energy dispersion spectroscopy analyses. It was also shown that the two phases in the eutectic were separated by thin boron interface and formed regular network of the Josephson junctions which very effectively absorbed microwave energy.
\end{abstract}

PACS numbers: 68.37.Yz, 74.10.+v, 74.25.Ha, 74.25.Nf, 74.50.+r

\section{Introduction}

Studies of inhomogeneous eutectic systems in which superconducting and matrix phases form lamellar structures have begun in the early sixties and still are of considerable interests. Only recently, Hooper et al. [1] reported on the $3 \mathrm{~K}$ phase in $\mathrm{Ru}-\mathrm{Sr}_{2} \mathrm{RuO}_{4}$ eutectic, which exhibited anomalous asymmetric features in $I-V$ characteristics due to breaking of time reversal symmetry. The matrix phase, which can be normal metal, another superconductor, semiconductor or dielectric strongly influence basic characteristics of superconducting

*corresponding author; e-mail: and@ifmpan.poznan.pl 
eutectic-like type of superconductivity, critical temperature and fields, flux pinning, and critical currents [2]. Properties also depend on geometry and laminar spacing of an eutectic structure. In general, there are two extreme behaviours: properties of eutectic can be a more or less simple sum of the properties of the two phases or it can behave as a completely homogeneous system due to proximity effect. An example of the first case is In-Bi system studied by Currie et al. [3], whereas Sn-Zn eutectic reported by Lutes and Clayton [4] behaved like a continuous material with decreasing critical temperature due to the proximity effect. Dobrosavljevic et al. [5] observed the effect of proximity on rf surface permeability of $\mathrm{Cd}-\mathrm{Sn}$ eutectic with superconducting and normal SNSN... lamellas. The lamellar structure can also form a regular network of the Josephson junctions, where properties of an eutectic system are controlled by tunnelling of the Cooper pairs. This case was studied by Isakov [6], who found strong geometry effects and resonant tunnelling across the Josephson contacts in $\mathrm{GaSb}-\mathrm{V}_{2} \mathrm{Ga}_{5}$ semiconductor-superconductor eutectic. In the present article we report on superconductivity in $\mathrm{Mo}_{2} \mathrm{Re}_{3} \mathrm{~B}_{x}-\mathrm{Mo}_{3} \mathrm{Re}_{2} \mathrm{~B}_{x}$ eutectic system composed of new, previously unknown superconducting phases. This system belongs to boride compounds, which are known to exhibit a strong electron-phonon interaction and high frequency vibration of lighter elements promoting enhanced critical temperatures. In ternary boride compounds, superconductivity has been recently discovered in similar phases, namely in $\mathrm{Mo}_{7} \mathrm{Re}_{13} \mathrm{~B}$ and $\mathrm{W}_{7} \mathrm{Re}_{13} \mathrm{~B}$ by Kawashima et al. $[7,8]$.

\section{Experimental}

The $\mathrm{Mo}_{2} \mathrm{Re}_{3} \mathrm{~B}_{x}-\mathrm{Mo}_{3} \mathrm{Re}_{2} \mathrm{~B}_{x}$ eutectic was synthesized by means of induction melting of the constituent elements in a water-cooled boat and under an argon atmosphere. The amounts of Mo, Re, and B fine powders with molar ratios of 7:13:1 and a total mass about $1 \mathrm{~g}$ were used for the melting. The ingot was inverted and remelted several times. In spite of this procedure, we found an excess of rhenium at the surface of the ingot caused by the nonstoichiometry between initial molar ratios of substrates and final composition of the eutectic. Thus, to ensure good homogeneity of the investigated sample it was cut from the interior of the ingot. The microstructure and cationic compositions were analysed by means of energy dispersion spectroscopy (EDS) on a ZEISS Ultra 55 scanning electron microscope (SEM). The EDS analysis did not confirm the formation of the $\mathrm{Mo}_{7} \mathrm{Re}_{13} \mathrm{~B}$ phase in the sample reported earlier by Kawashima [8].

Magnetic measurements were performed by means of Quantum Design MPMS SQUID magnetometer and also by an Oxford Instruments Ltd. MagLab 2000 System. For the electric transport measurements, a Quantum Design PPMS $7 \mathrm{~T}$ bridge and a standard four-point contact method were used. The measurements of magnetically modulated microwave absorption (MMMA) were performed using an ESR X-band $9.4 \mathrm{GHz}$ spectrometer equipped with a rectangular microwave 
cavity and Oxford Instruments Ltd. ESR 900 helium-flow cryostat. The standard electromagnet of the spectrometer was replaced by a set of the Helmholtz coils to provide a precise sweep of the DC magnetic field and to cancel earth magnetic field. The application of a second modulation of DC magnetic field with a frequency equal to $100 \mathrm{kHz}$ caused that the recorded signal was an electronic derivative of microwave absorption. The details concerning the method and the experimental setup can be found elsewhere $[9,10]$.

\section{Results and discussion \\ 3.1. Microstructure of the eutectic}

The SEM micrography in Fig. 1 presents the polished surface of the $\mathrm{Mo}_{2} \mathrm{Re}_{3} \mathrm{~B}_{x}-\mathrm{Mo}_{3} \mathrm{Re}_{2} \mathrm{~B}_{x}$ sample. Besides large grains of grey phase with dimensions of a few tenth of $\mu \mathrm{m}$ there are also regions composed of lamellas of two phases, namely, grey and black ones. Lamellas of these phases form a pattern characteristic of eutectics. These eutectic regions exhibit similarity to granular superconducting systems composed of isolated grains embedded in a normal matrix.

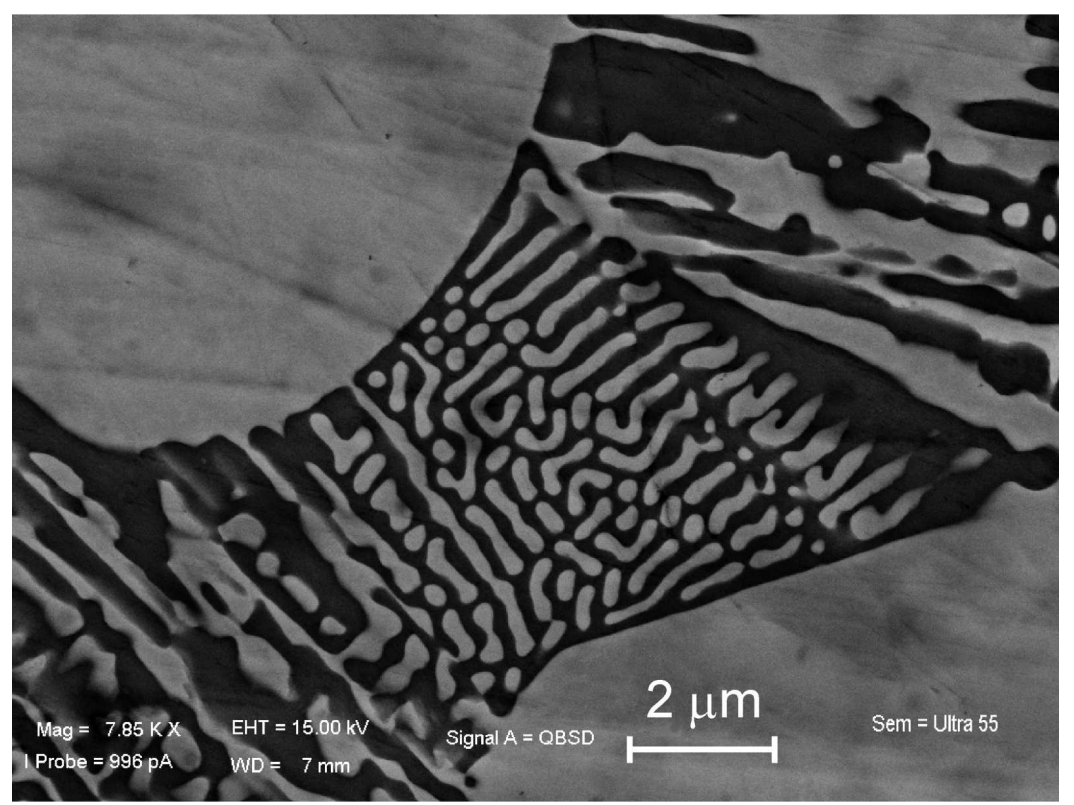

Fig. 1. The SEM micrography of $\mathrm{Mo}_{2} \mathrm{Re}_{3} \mathrm{~B}_{x}-\mathrm{Mo}_{3} \mathrm{Re}_{2} \mathrm{~B}_{x}$ sample.

An eutectic system is formed during solidification of an initial melt when two phases present in the melt do not form a solution for any relative concentration. Here, the melt was slightly shifted out of the optimal composition because the grey phase dominates in the alloy. Indeed, the even contents of the $\mathrm{Mo}_{2} \mathrm{Re}_{3} \mathrm{~B}_{x}$ 


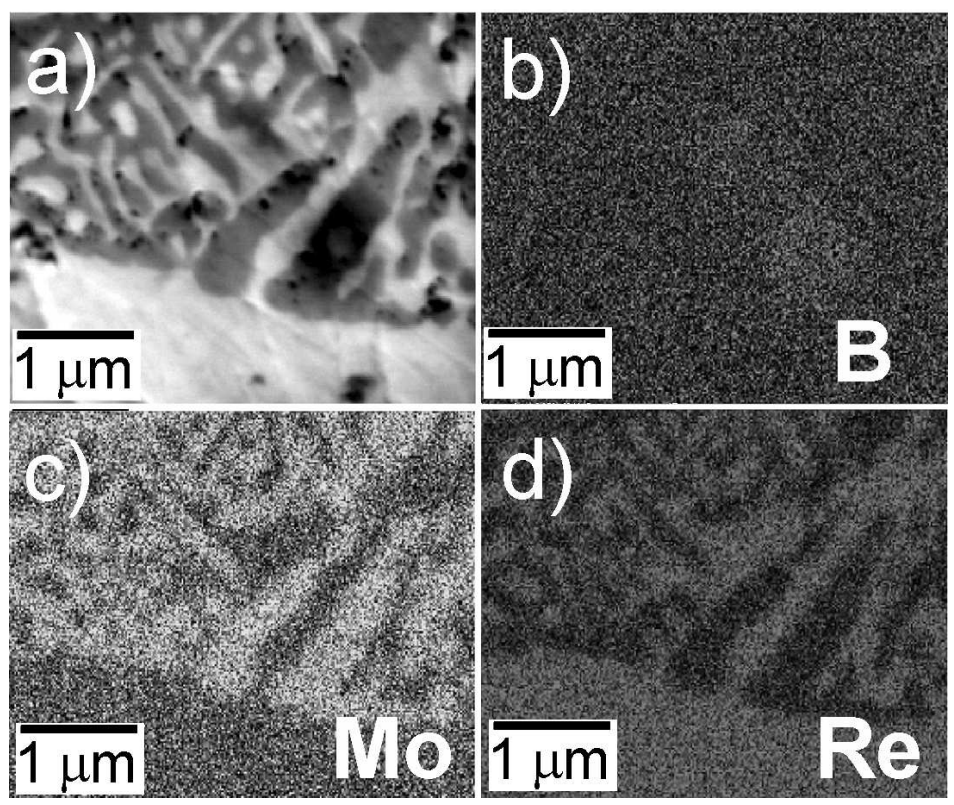

Fig. 2. SEM micrography of a selected region of the sample (a) and EDS distribution maps of B (b), Mo (c), and Re (d).

and $\mathrm{Mo}_{3} \mathrm{Re}_{2} \mathrm{~B}_{x}$ phases in the eutectic should be obtained for Mo:Re:B molar ratio equal to $5: 5: 2 \mathrm{x}$, which is rather far from the initial ratio of substrates equal to 7:13:1. The different volumes of the two phases allow for the eutectic to adjust its final composition to the initial substrates amount used for the synthesis. However, we found also an excess of unreacted rhenium deposited at the surface of the ingot as it was discussed in the experimental section. The exact composition of the two phases in eutectic was determined by means of EDS. Figure 2a presents the selected sample region for EDS analysis, and the spatial distributions of the elements are shown in Fig. 2b-d. In Fig. $2 \mathrm{~b}$ it is evident that the atoms of boron are distributed quite homogeneously in the sample. The dark regions in Fig. 2c indicate that the content of Mo is higher in the grey phase than in the black one. For Re the situation is opposite since the black phase exhibits a higher content of rhenium than the grey phase. Based on the energy spectrum and the relative intensities of X-ray radiation, the chemical compositions of the two phases were determined. The grey phase turned out to be $\mathrm{Mo}_{3} \mathrm{Re}_{2} \mathrm{~B}_{x}$ compound and the black phase $-\mathrm{Mo}_{2} \mathrm{Re}_{3} \mathrm{~B}_{x}$. We found no trace of $\mathrm{Mo}_{7} \mathrm{Re}_{13} \mathrm{~B}$ phase in any part of the sample despite using for the synthesis identical molar ratios of the constituent elements like it was reported by Kawashima for $\mathrm{Mo}_{7} \operatorname{Re}_{13} \mathrm{~B}$ [8] compound. The differences in final products are probably due to various thermal conditions during the alloying process. It seems that the synthesis in lower temperature by means of induction melting applied in the present case promotes the formation of the 
$\mathrm{Mo}_{2} \mathrm{Re}_{3} \mathrm{~B}_{x}-\mathrm{Mo}_{3} \mathrm{Re}_{2} \mathrm{~B}_{x}$ eutectic, whereas arc melting at high temperatures used by Kawashima generates the $\mathrm{Mo}_{7} \mathrm{Re}_{13} \mathrm{~B}$ compound. However, Kawashima in his report did not test microstructure of his samples.

The accurate determination of B amount was not possible since the energy of the boron line is too small, and the dimensions of black lamellas are comparable or lower to the spatial resolution of the electron beam. Also, at interfaces between the two phases, one can expect inhomogeneous distribution of boron. This point will be discussed later, in the part devoted to electric transport properties of this system.

\subsection{Properties of the eutectic}

Figure 3 shows details of magnetic superconducting transition. Here, the diamagnetic moment appears below the temperature of $6.6 \mathrm{~K}$. The transition is quite sharp and the transition width determined at $50 \%$ of diamagnetic signal is equal to $0.4 \mathrm{~K}$. A more careful inspection of the transition reveals that there is another drop of magnetic signal at higher temperature equal to $8.7 \mathrm{~K}$ (see the inset of Fig. 3). The complex structure of superconducting transition is much more clear in resistivity dependence on temperature presented in the inset of Fig. 4. The first step in resistivity occurs at a temperature of $8.9 \mathrm{~K}$ comparable to the beginning of the magnetic transition at $8.7 \mathrm{~K}$. The sample loses resistivity at a temperature of $6.4 \mathrm{~K}$, which corresponds well to a temperature of $6.6 \mathrm{~K}$ of a sharp drop in magnetic moment. Between the two above-mentioned temperatures, there is also another feature at $7.9 \mathrm{~K}$ followed by a gradual decrease in resistivity. The multistage character of superconducting transition in magnetic and electric properties can be associated with the presence of two superconducting phases in the system. The transition at higher temperature equal to $8.7 \mathrm{~K}$ in magnetic

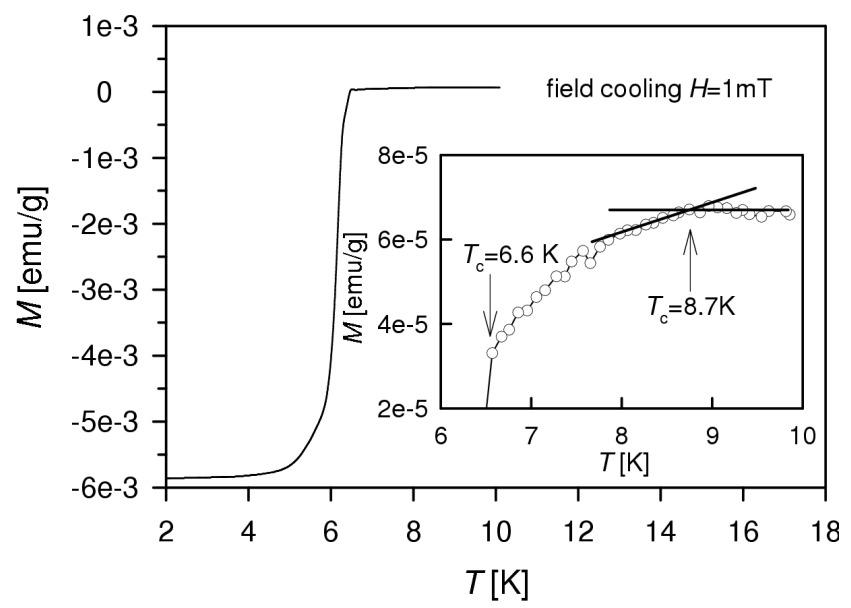

Fig. 3. The field cooled magnetic superconducting transition of the eutectic system. The applied DC magnetic field was equal to $1 \mathrm{mT}$. 


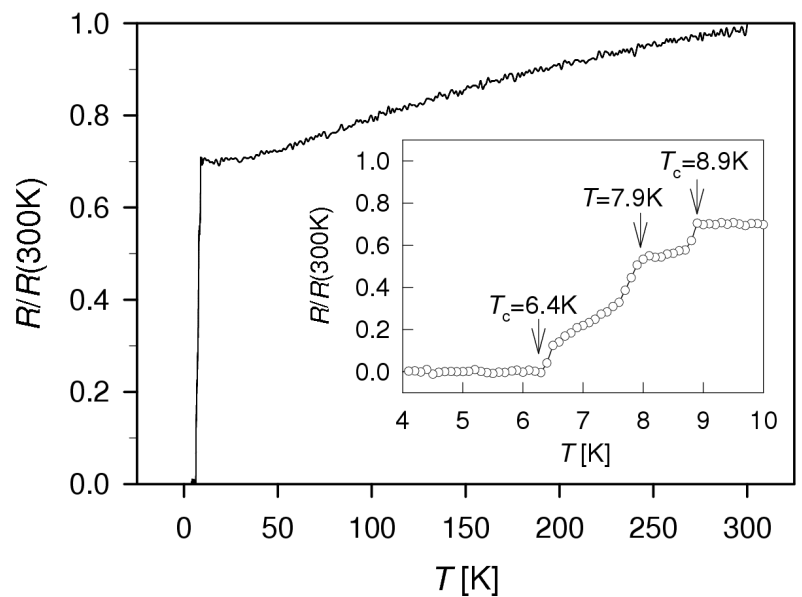

Fig. 4. The dependence of normalized resistivity in temperature (main part) and resistive superconducting transition (inset).

(or $8.9 \mathrm{~K}$ in transport) properties, should be related to the phase characterized by a small volume, namely to the lamellas of $\mathrm{Mo}_{2} \mathrm{Re}_{3} \mathrm{~B}_{x}$ compound. The arguments for this interpretation are as follows. In the magnetic properties, the superconducting transition of $\mathrm{Mo}_{2} \mathrm{Re}_{3} \mathrm{~B}_{x}$ phase is almost unnoticeable because of its negligible volume or due to the penetration of a magnetic flux into $\mathrm{Mo}_{2} \mathrm{Re}_{3} \mathrm{~B}_{x}$ black lamellas. The latter mechanism very effectively reduces a diamagnetic signal especially when dimensions of lamellas are comparable or smaller than a penetration depth $\lambda$. In transport properties, the superconducting transition in the lamellar system shunts electrically large volume of the sample and seriously suppresses the resistivity. On the other hand, the superconducting transition in large volume of grey $\mathrm{Mo}_{3} \mathrm{Re}_{2} \mathrm{~B}_{x}$ phase should lead to a sudden appearance of a diamagnetic signal and should also shunt totally the sample and lowers the resistivity to zero. Thus, the transition at $6.6 \mathrm{~K}$ in magnetic properties (or $6.4 \mathrm{~K}$ in transport) can be ascribed to the $\mathrm{Mo}_{3} \mathrm{Re}_{2} \mathrm{~B}_{x}$ dominant phase. The kink and decrease in electrical resistivity appearing at $7.9 \mathrm{~K}$ is probably associated with electrical percolation which takes place at this temperature in $\mathrm{Mo}_{2} \mathrm{Re}_{3} \mathrm{~B}_{x}$ lamellas. Indeed, $\mathrm{Mo}_{2} \mathrm{Re}_{3} \mathrm{~B}_{x}$ lamellas embedded in the $\mathrm{Mo}_{3} \mathrm{Re}_{2} \mathrm{~B}_{x}$ matrix, which is normal above $6.6 \mathrm{~K}$, can be regarded as a granular superconducting system. For percolating granular systems there are always two superconducting transitions [11-13]. First, at higher temperature a set of grains undergoes into superconducting state, next the set percolates and the grains form larger superconducting clusters at lower temperature. Therefore, the eutectic composed of $\mathrm{Mo}_{2} \mathrm{Re}_{3} \mathrm{~B}_{x}$ lamellas and of continuous $\mathrm{Mo}_{3} \mathrm{Re}_{2} \mathrm{~B}_{x}$ matrix, should exhibit three superconducting transitions; two for the set of lamellas and one for the matrix. We rather exclude other explanation in terms of the proximity effect because the two phases possess independent superconducting transitions and the whole eutectic behaves like an inhomogeneous system [3]. The resistivity of 
the eutectic in the wide temperature range is shown in the main part of Fig. 4 . In general, the resistivity of the sample is rather high as for metals and it decreases with temperature very gradual. The residual resistivity ratio (RRR) defining temperature variations of resistivity as $\mathrm{RRR}=R(10 \mathrm{~K}) / R(300 \mathrm{~K})$ is high and equal to 0.7 . This phenomenon is due to nonstoichiometry and boron excess in interfaces between the two phases. The semiconducting layer of boron deposited in interfaces increases significantly the resistivity of the system and makes it almost independent of temperature. A similar behaviour was found in $\mathrm{W}_{7} \mathrm{Re}_{13} \mathrm{~B}$ and $\mathrm{Mo}_{7} \mathrm{Re}_{13} \mathrm{~B}$ and was explained in the same way $[7,8]$.

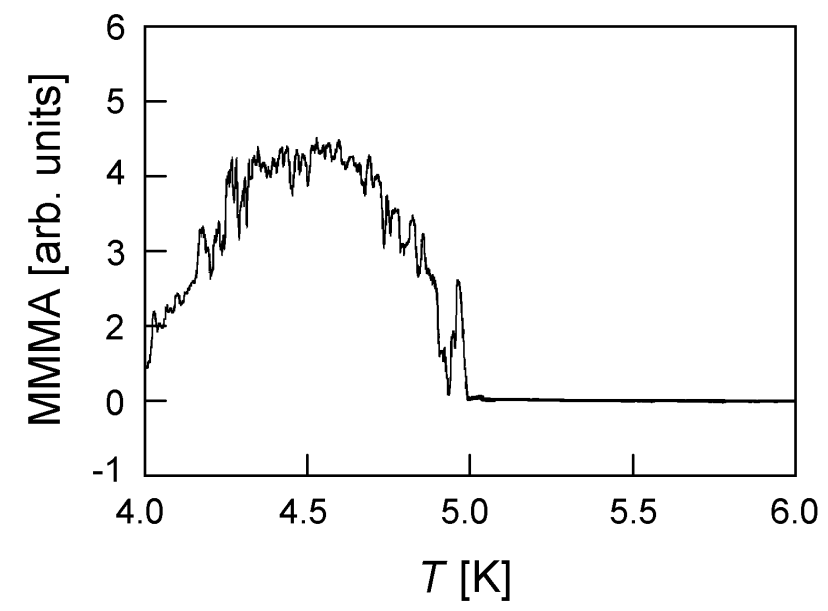

Fig. 5. MMMA signal versus temperature recorded in $\mathrm{Mo}_{2} \mathrm{Re}_{3} \mathrm{~B}_{x}-\mathrm{Mo}_{3} \mathrm{Re}_{2} \mathrm{~B}_{x}$ eutectic.

In the eutectic regions composed of two superconducting phases $\mathrm{Mo}_{2} \mathrm{Re}_{3} \mathrm{~B}_{x}$ and $\mathrm{Mo}_{3} \mathrm{Re}_{2} \mathrm{~B}_{x}$ separated by semiconducting layer of boron, there exists a Josephson junction network. Besides numerous phenomena originating from tunnelling of the Cooper pairs, the Josephson junctions characterize strong absorption of energy in microwave band [14-17]. Therefore, the $\mathrm{Mo}_{2} \mathrm{Re}_{3} \mathrm{~B}_{x}-\mathrm{Mo}_{3} \mathrm{Re}_{2} \mathrm{~B}_{x}$ eutectic exhibits very large MMMA which is comparable in magnitude to the one observed in granular high-temperature superconductors. Figure 5 shows MMMA dependence on temperature. The MMMA signal appears at the temperature equal to $5 \mathrm{~K}$, i.e. more than $1 \mathrm{~K}$ below the main transition in the eutectic sample. It is a common behaviour of microwave absorption in granular systems because the Josephson junctions become superconducting at lower temperature than the one of grains [11]. Junctions suffer also from a spread of transition temperatures. On further cooling, the MMMA signal starts to increase, attains the maximum and next gradually decreases. This feature can be also modelled in terms of vortices penetration towards the system of the Josephson junctions characterized by various parameters [18-20]. 


\section{Conclusions}

We synthesized and investigated a new superconducting eutectic composed of previously unreported superconducting phases, namely of $\mathrm{Mo}_{2} \mathrm{Re}_{3} \mathrm{~B}_{x}$ and of $\mathrm{Mo}_{3} \mathrm{Re}_{2} \mathrm{~B}_{x}$ with boron content $x \approx 1$. The phases were identified by means of EDS analyses. The eutectic was an inhomogeneous system with two distinct critical temperatures equal to $6.6 \mathrm{~K}$ and $8.7 \mathrm{~K}$ (or $6.4 \mathrm{~K}$ and $8.9 \mathrm{~K}$ in transport measurements) corresponding to $\mathrm{Mo}_{3} \mathrm{Re}_{2} \mathrm{~B}_{x}$ and $\mathrm{Mo}_{2} \mathrm{Re}_{3} \mathrm{~B}_{x}$ phase, respectively. There was no evidence of the proximity effect. In the microstructure of the sample there were eutectic regions with regular network of the Josephson junctions. The junctions were naturally formed by superconducting lamellas separated by semiconducting layer of boron due to nonstoichiometry and boron excess at the interfaces between the two phases. Thus, the eutectic exhibited a strong microwave absorption, which was comparable in magnitude to that one observed in granular high-temperature superconductors.

\section{Acknowledgments}

B.A. acknowledges financial support from EGIDE foundation under ECO-NET 10188YD project.

\section{References}

[1] J. Hooper, Z.Q. Mao, K.D. Nelson, Y. Liu, M. Wada, Y. Maeno, Phys. Rev. B 70, 14510 (2004).

[2] J.D. Livingston, J. Appl. Phys. 38, 2408 (1967).

[3] P.D. Currie, T.R. Finlayson, W.A. Rachinger, Scr. Metall. 11, 59 (1977).

[4] O.S. Lutes, D.A. Clayton, Phys. Rev. 145, 218 (1966).

[5] L. Dobrosavljevic, C. Petipas-Dupuis, R. Racek, Phys. Status Solidi 38, 159 (1970).

[6] G.I. Isakov, Inorg. Mater. 39, 1117 (2003).

[7] K. Kawashima, A. Kawano, T. Muranaka, J. Akimitsu, J. Phys. Soc. Jpn. 74, $700(2005)$.

[8] K. Kawashima, A. Kawano, T. Muranaka, J. Akimitsu, Physica B 378-380, 1118 (2006).

[9] J. Stankowski, B. Czyżak, J. Martinek, B. Andrzejewski, in: Recent Developments in High Temperature Superconductivity, Eds. J. Klamut, B.W. Veal, B.M. Dabrowski, P.W. Klamut, E. Kazimierski, Springer, Berlin 1996, p. 167.

[10] J. Stankowski, B. Andrzejewski, B. Czyżak, W. Hilczer, Physica C 215, 167 (1993).

[11] E. Guilmeau, B. Andrzejewski, J.G. Noudem, Physica C 387, 382 (2003).

[12] E. Guilmeau, B. Andrzejewski, G. Desgardin, Physica C 377, 304 (2002).

[13] J.R. Clem, Physica C 153, 50 (1988).

[14] J. Stankowski, P.K. Kahol, N.S. Dalal, J.S. Moodera, Phys. Rev. B 36, 7126 (1987). 
[15] K.W. Blazey, K.A. Müller, J.G. Bednorz, W. Berlinger, G. Amoretti, E. Buluggiu, A. Vera, F.C. Matacotta, Phys. Rev. B 36, 7241 (1987).

[16] R. Durny, J. Hautala, S. Ducharme, B. Lee, O.G. Symko, P.C. Taylor, D.J. Zheng, J.A. Xu, Phys. Rev. B 36, 2361 (1987).

[17] B. Andrzejewski, A. Kowalczyk, J. Stankowski, A. Szlaferek, J. Phys. Chem. Solids 65, 623 (2004).

[18] B. Czyżak, Physica C 243, 327 (1995).

[19] B. Czyżak, Supercond. Sci. Technol. 9, 149 (1996).

[20] A. Rycerz, J. Spałek, Physica C 387, 97 (2003). 